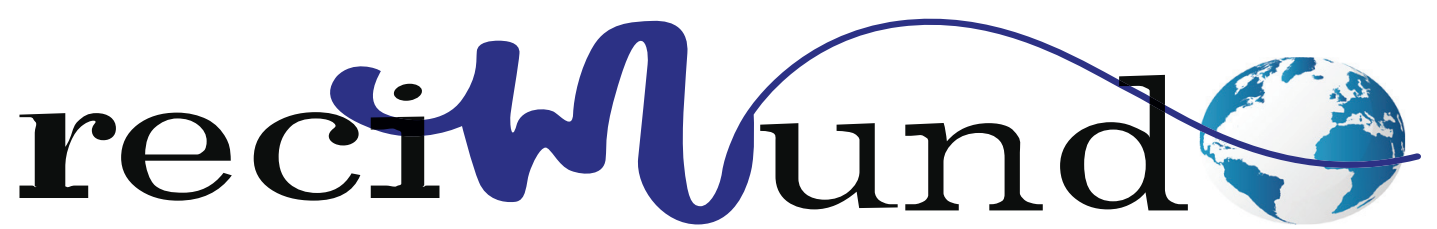

Revista Científica Mundo de la Investigación y el Conocimiento

DOI: 10.26820/recimundo/4.(1).esp.marzo.2020.182-190

URL: http://recimundo.com/index.php/es/article/view/780

EDITORIAL: Saberes del Conocimiento

REVISTA: RECIMUNDO

ISSN: 2588-073X

TIPO DE INVESTIGACIÓN: Artículo de Revisión

CÓDIGO UNESCO: 5801 Teoría y Métodos Educativos

PAGINAS: $182-190$

\title{
Aprendizaje inclusivo para las personas con discapacidad
}

\author{
Inclusive learning for people with disabilities
}

Os tiques como uma ferramenta para gerenciamento de riscos

\section{Douglas Vicente Vásquez Aguilar'; Luis Enrique Soto Chávez²; Maria Allauca Amaguaya3; Fausto Arturo Benitez Troya ${ }^{4}$}

\section{RECIBIDO: 20/11/2019 ACEPTADO: 29/01/2020 PUBLICADO: 05/03/2020}

1. Magister en Diseño Curricular; Diploma Superior en Pedagogía Universitaria; Licenciado en Ciencias Sociales y Polóticas; Abogado de los Tribunales y Juzgados de la República del Ecuador; Docente Facultad Ingeniería Industrial; Universidad de Guayaquil; Guayaquil, Ecuador; douglas.vasqueza@hotmail.com; (D) https://orcid. org/0000-0003-2403-6916

2. Magister Universitario en Ingenieria Avanzada de Producción Logística y Cadena de Suministro; Ingeniero Industrial; Docente Facultad Ingeniería Industrial; Universidad de Guayaquil; Guayaquil, Ecuador; luis.sotoc@. ug.edu.ec; (D) https://orcid.org/0000-0001-8854-6881

3. Magister en Psicología Laboral con Mención en Desarrollo Humano y de Organización; Magister en Seguridad, Higiene Industrial y Salud Ocupacional; Ingeniera en Marketing; Docente Facultad Ingeniería Industrial; Universidad de Guayaquil; Guayaquil, Ecuador; maria.allaucaam@ug.edu.ec ; (D) https://orcid.org/0000-00027399-489X

4. Magister en Finanzas y Proyectos Corporativos; Economista; Docente Facultad Ingeniería Industrial; Universidad de Guayaquil; Guayaquil, Ecuador; fausto.benitezt@ug.edu.ec; (D) https://orcid.org/0000-0003-4464-0176

CORRESPONDENCIA

Douglas Vicente Vásquez Aguilar

douglas.vasqueza@hotmail.com

Bolívar, Ecuador 
Este trabajo de investigación relacionado con el aprendizaje inclusivo para las personas con discapacidades, deberá incluirse en todas las mallas curriculares, sílabos, planes analíticos, de las diferentes universidades públicas y privadas del país, con el fin de no vulnerar los derechos Constitucionales que tienen las personas con discapacidad a la educación superior incluyente, además los docentes de educación superior tenemos que brindar todas las facilidades orientar o guiar a nuestros estudiantes con discapacidad para que ellos niño $y$ ad niños y adolescentes, mujeres embrazadas, personas con díncapacidad, personas privadas de libertad y quienes adolezcan de en recibirán atención prioritaria y especializada en los ambitos público y privado, la misma atención prioritaria recibirañ las personas en situación de riesgo, las víctimas de violencia

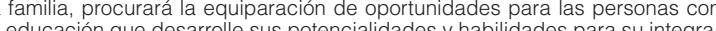

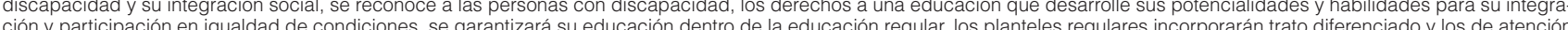
ción y participación en igualdad de condiciones, se garantizara su educacion dentro de la educación regular, los planteles regulares incorporarán trato diferenciado y los de atención especial la educación especializada. los establecimientos educativos cumplirán normas de accesibilidad para personas con discapacidad e implementarán un sistema de becas que responda a las condiciones económicas de este grupo, la educación especializada para las personas con discapacidad intelectual y el fomento de sus capacidades mediante la creación de centros educativos y programas de enseñanza específicos, 48, el estado adoptará a favor de las personas con discapacidad medidas que aseguren, la obtención de créditos y rebajas o exoneraciones tributarias que les permita iniciar y mantener actividades productivas, y la obtención de becas de estudio en todos los niveles de educación, Ley Nacional de Educación y del Sistema de Educación Superior, sus estudios, para obtener educación, formación y/o capacitación, asistiendo a clases en un establecimiento educativo especializado o en un establecimiento de educación escolarizada, según el caso, Artículo 28. educación inclusiva, la autoridad educativa nacional implementará las medidas pertinentes, para promover la inclusión de estudiantes con necesidades educativas especiales que requieran apoyos tecnico tecnológicos y humanos, tales como personal especializado, temporales o permanentes y/o adaptaciones curriculares y de accesibilidad física, comunicacional y espacios de aprendizaje, en un establecimiento de educación escolarizada, para atención de personas con necesidades educativas especiales, con énfasis en sugerencias pedagógicas para la atención educativa a cada tipo de discapacidad. esta normativa será de cumplimiento obligatorio para todas las instituciones educativas en el Sistema Educativo Nacional, Artículo 38 . Becas, aquellas personas con discapacidad en cuya localidad no de cumplimiento obligatorio para todas las instituciones educativas en el Sistema Educativo Nacional, Artículo 38. Becas, aquellas personas con discapacidad en cuya localidad no
exista un establecimiento educativo público con servicios adecuados para atender a sus necesidades educativas especiales podrán recibir del Instituto Ecuatoriano de Crédito Educativo, becas y créditos educativos, a fin de que asistan a una institución educativa particular o fisco misional que sí ofrezca los servicios adecuados, de conformidad con la normativa especifica que se expida para el efecto, Artículo 38 , Becas para aquellas personas con discapacidad en cuya localidad no exista un establecimiento educativo público con servicios adecuados para atender a sus necesidades educativas especiales podrán recibir del Instituto Ecuatoriano de Crédito Educativo, becas y créditos educativos, a fin de que asistan a una institución educativa particular o fisco misional que sí ofrezca los servicios adecuados, de conformidad con la normativa específica que se expida para el efecto, la Secretaria (n) nivel, en sus modalidades presencial, semipresencial y a distancia, para personas con discapacidad, aplicando criterios de equidad de género, Artículo 40 , difusión en el ámbito de educación superior, la Secretaría Nacional de Educación Superior, Ciencia, Tecnología e Innovación, asegurará que en todas las instituciones de educación superior se transversalice el conocimiento del tema de la discapacidad dentro de las mallas curriculares de las diversas carreras y programas académicos, dirigidos a la inclusión de las personas con discapacidad y a la formación humana de las y los futuros profesionales.

Palabras clave: Aprendizaje, Inclusivo, Personas, Discapacidad

\section{ABSTRACT}

This research work related to inclusive learning for people with disabilities, should be included in all curricular meshes, syllables, analytical plans, of the different public and private universities of the country, in order not to violate the Constitutional rights that have the people with disabilities to inclusive higher education, in addition to higher education teachers we have to provide all facilities to guide or guide our students with disabilities so that they receive priority attention in their learning process, as indicated by our Constitution of the Republic of Ecuador in its articles, 35 older adults, girls, boys and adolescents, pregnant women, people with disabilities, persons deprived of liberty and those suffering from catastrophic or highly complex diseases, will receive priority and specialized attention in the public and private, the same priority attention will be given to people at risk, victims of domestic and sexual violence, child abuse, natural or anthropogenic disasters., the State will provide special protection to persons in a condition of double vulnerability, 47 , the State will guarantee policies of prevention of disabilities and, jointly with society and family, will seek to equalize opportunities for people with disabilities and their social integration, persons with disabilities are recognized, the rights to an education that develops their potential and abilities for their integration and participation in equal conditions, their education will be guaranteed within the regular education, the regular schools will incorporate differentiated treatment and those of special attention the specialized education. The educational establishments will comply with accessibility standards for people with disabilties and wil implement a scholarship system that responds to the economic conditions of this group, specialized education for people with intellectual disabilities and the development of their capacities through the creation of educational centers and programs Specific education, 48 , the state will adopt measures to ensure Deople with disabilies, obtaining credits and tax rebates or exemptions that allow them to initiate and maintain productive activities, and obtaining scholarships at all levels of education, Organic Law on Disabilities, Article 27, right to education the State shall ensure that persons with disabilites can access, remain and complete, within the National Educalion System (a) establishment, as appropriate, Article 28. Inclusive education, the national educational authorly shall implement the pertinent measures, to promote the inclusion of students with special ( cationa and learling spaces, in a schol edulis naliona in whos to each type of dlsabily. This regulation will be mandatory for all educational instititions in the National Education System, A

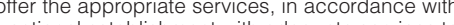
the specilic regulations issued for this purpose, Article 38, Scholarships for those with Disability in whose locality there is no public educational establishment with adequate services to institution or missionary treasury that does offer to the appropriate services, in accordance with the specific regulations issued for this purpose, the National Secretariat of Higher Education, Science, Technology and Innovation will enforce the granting of third and fourth level scholarships to public and private higher education institutions.

Keywords: Learning, Inclusive, People, Disability.

\section{RESUMO}

Este trabalho de pesquisa relacionado à aprendizagem inclusiva para pessoas com deficiência deve ser incluído em todas as malhas curriculares, sílabas, planos analíticos das diferentes universidades públicas e privadas do país, a fim de não violar os direitos constitucionais que têm as pessoas com deficiência ao ensino superior inclusivo, além dos professores de ensino superior, temos que fornecer todas as facilidades para orientar ou orientar nossos alunos com deficiência, para que eles recebam atenção prioritária em seu processo de a ensizagem, deficiência pessoas privadas de liberdade e pessoas que sofrem de doenças catastróficas ou de alta complexidade, receberão atenção prioritária e especializada em público e privado, a mesma atenção prioritária será dada às pessoas em risco, vítimas de violência doméstica e sexual, abuso infantil, natural ou antropogênico catástrofes políticas., o Estado proporcionará proteção especial às pessoas em condição de dupla vulnerabilidade, 47, o Estado garantirá políticas de prevenção de deficiências e, em conjunto com a sociedade e a família, buscará equalizar as oportunidades para as pessoas com deficiência e seus interesses sociais. integração, pessoas com deficiência são reconhecidas, direitos a uma educação que desenvolva seu potencial e habilidades para sua integração e participação em igualdade de condições, sua educação será garantida dentro da educação regular, as escolas regulares incorporarão tratamento diferenciado e atenção especial a educação especializada. Os estabelecimentos de ensino cumprirão os padrões de acessibilidade para pessoas com deficiência e implementarão um sistema de bolsas de estudo que responda às condições econômicas desse grupo, educação especializada para pessoas com deficiência intelectual e desenvolvimento de suas capacidades através da criação de centros e programas educacionais. educação, 48, o estado adotará medidas para garantir às pessoas com deficiência, obter créditos e abatimentos ou isenções de impostos que lhes permitam iniciar e manter atividades produtivas e obter bolsas de estudos em todos os níveis de educação, Lei Orgânica da Deficiência, Artigo 27, à educacão, o Estado garantirá que as pessoas com deficiência possam acessar permanecer e concluir dentro do Sistema Nacional de cação, Lei Orgânica da Deficiência, Artigo 27, a educação, o Estado garantiră que as pessoas com deficiência possam acessar, permanecer e concluir, dentro do Sistema Nacional de zado ou num estabelecimento de ensino escolar, como Art. 28. Educação inclusiva, a autoridade educacional nacional implementará as medidas pertinentes para promover a inclusão de estudantes com necessidades educacionais especiais que exijam apoio técnico e tecnológico humano, como equipe especializada, temporária ou permanente e / ou adaptações curriculares e espaços físicos, comunicacionais e de aprendizagem, em um estabelecimento de educação escolar, para esse fim, a autoridade educacional nacional formulará, emitirá e supervisionará a conformidade com os regulamentos nacionais que serão atualizados a cada ano e incluirá diretrizes para o atendimento de pessoas com necessidades educacionais especiais, com ênfase em sugestões pedagógicas para atenção educacional a cada tipo de deficiência. Este regulamento será obrigatório para todas as instituições de ensino do Sistema Nacional de Educação, artigo 38. Bolsas de estudo, aquelas com deficiência em cuja localidade não existe estabelecimento educacional público com serviços adequados para atender às suas necessidades educacionais especiais, podem receber do Crédito Educacional do Instituto Equatoriano, bolsas de estudo e créditos educacionais, para que atendam a uma instituição educacional ou tesouraria missionária específica que ofereça os serviços adequados, de acordo com os regulamentos específicos emitidos para esse fim. Artigo 38, Bolsas de estudo para pessoas com deficiência em cuja localidade não exista educação pública. O estabelecimento com serviços adequados para atender às suas necessidades educacionais especiais poderá receber do Instituto Equatoriano de Crédito Educacional, bolsas de estudo e créditos educacionais, para frequentar uma instituição educacional ou um tesouro missionário que ofereça os serviços adequados, de acordo com as especificações Com regulamentos específicos emitidos para esse fim, a Secretaria Nacional de Educação Superior, Ciência, Tecnologia e Inovação aplicará a concessão de bolsas de terceiro e quarto nível para instituições de ensino superior públicas e privadas. 


\section{Introducción}

En Ecuador la educación superior deberá ser inclusiva que brinde todas las facilidades a los estudiantes con discapacidades, desde el punto de vista académico obtenido por el docente en el desarrollo de sus actividades diarias y permanentes en el aula de clases se optimiza el conocimiento entre el profesor y los estudiantes con discapacidad el docente un ffacilitador o guía que deberá otorgarle todas las herramientas didácticas pedagógicas para el aprendiente pueda fortalecer sus conocimientos académicos y continuar con su proceso de estudios superiores, con el objetivo formar excelentes profesionales técnicos en el área de la Ingeniería Industrial que su discapacidad no es ningún obstáculo para la superación académica, 47, el Estado garantizará políticas de prevención de las discapacidades y, de manera conjunta con la sociedad y la familia, procurará la equiparación de oportunidades para las personas con discapacidad y su integración social, se reconoce a las personas con discapacidad, los derechos a una educación que desarrolle sus potencialidades y habilidades para su integración y participación en igualdad de condiciones, se garantizará su educación dentro de la educación regular, los planteles regulares incorporarán trato diferenciado y los de atención especial la educación especializada. Ios establecimientos educativos cumplirán normas de accesibilidad para personas con discapacidad e implementarán un sistema de becas que responda a las condiciones económicas de este grupo, la educación especializada para las personas con discapacidad intelectual y el fomento de sus capacidades mediante la creación de centros educativos y programas de enseñanza específicos, 48 , el estado adoptará a favor de las personas con discapacidad medidas que aseguren, la obtención de créditos y rebajas o exoneraciones tributarias que les permita iniciar y mantener actividades productivas, y la obtención de becas de estudio en todos
Ios niveles de educación, Ley Orgánica de Discapacidades, Artículo 27, derecho a la educación el Estado procurará que las personas con discapacidad puedan acceder, permanecer y culminar, dentro del Sistema Nacional de Educación y del Sistema de Educación Superior, sus estudios, para obtener educación, formación y/o capacitación, asistiendo a clases en un establecimiento educativo especializado o en un establecimiento de educación escolarizada, según el caso, Artículo 28. educación inclusiva, la autoridad educativa nacional implementará las medidas pertinentes, para promover la inclusión de estudiantes con necesidades educativas especiales que requieran apoyos técnico tecnológicos y humanos, tales como personal especializado, temporales o permanentes y/o adaptaciones curriculares y de accesibilidad física, comunicacional y espacios de aprendizaje, en un establecimiento de educación escolarizada, para el efecto, la autoridad educativa nacional formulará, emitirá y supervisará el cumplimiento de la normativa nacional que se actualizará todos los años e incluirá lineamientos para la atención de personas con necesidades educativas especiales, con énfasis en sugerencias pedagógicas para la atención educativa a cada tipo de discapacidad. esta normativa será de cumplimiento obligatorio para todas las instituciones educativas en el Sistema Educativo Nacional, Artículo 38. Becas, aquellas personas con discapacidad en cuya localidad no exista un establecimiento educativo público con servicios adecuados para atender a sus necesidades educativas especiales podrán recibir del Instituto Ecuatoriano de Crédito Educativo, becas y créditos educativos, a fin de que asistan a una institución educativa particular o fisco misional que sí ofrezca los servicios adecuados, de conformidad con la normativa específica que se expida para el efecto, Artículo 38, Becas para aquellas personas con discapacidad en cuya localidad no exista un establecimiento educativo público con servicios adecuados para atender a sus necesidades educati- 
vas especiales podrán recibir del Instituto Ecuatoriano de Crédito Educativo, becas y créditos educativos, a fin de que asistan a una institución educativa particular o fisco misional que sí ofrezca los servicios adecuados, de conformidad con la normativa específica que se expida para el efecto, la Secretaria Nacional de Educación Superior, Ciencia, Tecnología e Innovación hará cumplir a las instituciones de educación superior públicas y privadas la concesión de becas de tercer y cuarto nivel, en sus modalidades presencial, semipresencial y a distancia, para personas con discapacidad, aplicando criterios de equidad de género, Artículo 40, difusión en el ámbito de educación superior, la Secretaría Nacional de Educación Superior, Ciencia, Tecnología e Innovación, asegurará que en todas las instituciones de educación superior se transversalice el conocimiento del tema de la discapacidad dentro de las mallas curriculares de las diversas carreras y programas académicos, dirigidos a la inclusión de las personas con discapacidad y a la formación humana de las y los futuros profesionales.

\section{Aporte cognitivo}

\section{Aprendizaje inclusivo en la educación su- perior pública y privada en Ecuador}

Los docentes de educación superior tenemos que brindar todas las facilidades orientar o guiar a nuestros estudiantes con discapacidad para que ellos reciban atención prioritaria en su proceso de aprendizaje, así como lo señala nuestra Constitución de la República del Ecuador, además consideramos que desde nuestro punto de vista en calidad de docentes de la Facultad Ingeniería Industrial Universidad de Guayaquil y por nuestra propia experiencia en el dictado de clases por varios años consecutivos hemos observado que es necesario implementar el aprendizaje inclusivo el mismo que mejoraría el conocimiento de los estudiantes de la carrera Ingeniería Industrial, seguros estamos que por los resultados en los apren- dientes serán excelentes y se los realizaran mediante trabajos de exposiciones colectivas e individuales en primer, segundo parcial, así mismo permite la retro alimentación del conocimiento en la educación superior, aprende el estudiante y consecuentemente el profesor, en nuestras actividades diarias de docentes descubrimos que en el aula de clases con talleres o trabajos académicos prácticos interactuados se construye el conocimiento, por varias ocasiones nos tocó interactuar con brillantes estudiantes con discapacidad demostrando que tienen un coeficiente intelectual muy desarrollado, los profesores también aprenden de ellos, el proceso de enseñanza aprendizaje debe ser bilateral que permita descubrir, extraer el conocimiento del estudiante para ayudarlo sus fortalezas y debilidades a cumplir su objetivo ser competitivo, buscando siempre la excelencia académica, para brindar un aporte muy significativo en el desarrollo sostenible y sustentable e inclusivo en el Ecuador, el Docente de la universidad de Guayaquil en este siglo debe centrarse de manera directa en el estudiante fortaleciendo permanentemente el aprendizaje, en la praxis el desarrollo integral del conocimiento, con valores éticos y morales, impulsando continuamente la Innovación, basada en competencias, utilizando herramientas informáticas actualizadas que permitan desde nuestras cátedras y por intermedio de teleconferencias de forma directa observando clases en línea en diferentes universidades nacionales y extranjeras y básicamente de países de habla hispana, de esta manera fomentaremos la praxis en el aula de clases, utilizando la metodología inclusiva se aprende haciendo o practicando en talleres a desarrollarse en aula de clases.

\section{Métodos}

\section{Método Inductivo}

Se trata del método científico más usual, en el que pueden distinguirse cuatro pasos esenciales: la observación de los hechos 
para su registro; la clasificación y el estudio de estos hechos; la derivación inductiva que parte de los hechos y permite llegar a una generalización; y la contrastación.

\section{Método deductivo}

El método deductivo es un método científico que considera que la conclusión se halla implícita dentro las premisas. Esto quiere decir que las conclusiones son una consecuencia necesaria de las premisas: cuando las premisas resultan verdaderas y el razonamiento deductivo tiene validez, no hay forma de que la conclusión no sea verdadera.

\section{Método analítico}

El Método analítico es aquel método de investigación que consiste en la desmembración de un todo, descomponiéndolo en sus partes o elementos para observar las causas, la naturaleza y los efectos

\section{Resultados}

Con la implementación del nuevo modelo educativo denominado Aprendizaje Inclusivo los resultados son muy positivos y halagadores en el desarrollo del conocimiento de los estudiantes con discapacidad de diferentes asignaturas en la Facultad de Ingeniería Industrial de la Universidad de Guayaquil, se evidencian con las exposiciones colectivas e individuales y trabajos desarrollados por los estudiantes en primer y segundo parcial.

\section{Discusión}

Con el debate del tema aprendizaje Inclusivo y su aplicabilidad en el aula de clases, el mismo que se discutió con varios Docentes y aproximadamente ciento veinte estudiantes de la Facultad de Ingeniería Industrial de la Universidad de Guayaquil, se tomó la decisión de acoger, aportar e implantar este modelo educativo denominado Aprendizaje
Inclusivo el mismo que ayudaría en la superación academica de la educación superior inclusiva el Ecuador.

\section{Conclusiones}

En la universidad de Guayaquil y en cada una de sus unidades académicas de manera muy particular en la Facultad de Ingeniería Industrial, la educación superior en la actualidad impartida por sus profesores es de calidad, calidez, con valores éticos y morales, epistemológica, humanista, excelente e inclusiva y permite con absoluta libertad y confianza a los estudiante demostrar sus conocimientos, construidos en el aula de clases, su propio desarrollo académico por intermedio del método aprendizaje inclusivo para beneficio de las personas con discapacidades, como una herramienta diaria de trabajo en los espacios de aprendizaje, para los Docentes de Educación Superior en la Universidad de Guayaquil.

\section{Recomendación}

La Universidad de Guayaquil deberá elaborar un plan de formación, capacitación Epistemológica, Holística, Axiológica, Humanista e inclusiva permanente, como política Institucional, para que su claustro de Docente, fortalezca y actualice sus conocimientos de manera continua en el desarrollo de estudios superiores de posgrado para que mejoren sus competencias en el aprendizaje inclusivo de personas con discapacidades diferentes, con la actualización académica de sus Profesores, la Facultad de Ingeniería Industrial de la Universidad de Guayaquil obtendrá como resultado la Optimización del aprendizaje Inclusivo en todos sus estudiantes, consiguiendo como objetivo o producto final la obtención de brillante Ingenieros Industriales con competencias en el aprendizaje inclusivo de estudiantes con discapacidad. 


\section{Agradecimiento}

A Dios nuestro creador por otorgarnos la vida, salud, fuerza y el valor que nos brinda cada día de nuestra existencia para continuar con el trabajo y la lucha permanente hasta conseguir nuestro objetivo en calidad de Docentes de la Facultad Ingeniería Industrial Universidad de Guayaquil.

\section{Comentarios de los autores}

\section{(Asamblea Constituyente, 2008)}

Artículo 47, el Estado garantizará políticas de prevención de las discapacidades y, de manera conjunta con la sociedad y la familia, procurará la equiparación de oportunidades para las personas con discapacidad y su integración social, se reconoce a las personas con discapacidad, los derechos a una educación que desarrolle sus potencialidades y habilidades para su integración y participación en igualdad de condiciones, se garantizará su educación dentro de la educación regular, los planteles regulares incorporarán trato diferenciado y los de atención especial la educación especializada. los establecimientos educativos cumplirán normas de accesibilidad para personas con discapacidad e implementarán un sistema de becas que responda a las condiciones económicas de este grupo, la educación especializada para las personas con discapacidad intelectual y el fomento de sus capacidades mediante la creación de centros educativos y programas de enseñanza específicos, 48, el estado adoptará a favor de las personas con discapacidad medidas que aseguren, la obtención de créditos y rebajas o exoneraciones tributarias que les permita iniciar y mantener actividades productivas, y la obtención de becas de estudio en todos los niveles de educación. (Constitución de la República del Ecuador)

\section{(Asamblea Nacional, 2012)}

Artículo 27, derecho a la educación el Es- tado procurará que las personas con discapacidad puedan acceder, permanecer y culminar, dentro del Sistema Nacional de Educación y del Sistema de Educación Superior, sus estudios, para obtener educación, formación y/o capacitación, asistiendo a clases en un establecimiento educativo especializado o en un establecimiento de educación escolarizada, según el caso, Artículo 28. educación inclusiva, la autoridad educativa nacional implementará las medidas pertinentes, para promover la inclusión de estudiantes con necesidades educativas especiales que requieran apoyos técnico tecnológicos y humanos, tales como personal especializado, temporales o permanentes y/o adaptaciones curriculares y de accesibilidad física, comunicacional y espacios de aprendizaje, en un establecimiento de educación escolarizada, para el efecto, la autoridad educativa nacional formulará, emitirá y supervisará el cumplimiento de la normativa nacional que se actualizará todos los años e incluirá lineamientos para la atención de personas con necesidades educativas especiales, con énfasis en sugerencias pedagógicas para la atención educativa a cada tipo de discapacidad. esta normativa será de cumplimiento obligatorio para todas las instituciones educativas en el Sistema Educativo Nacional, Artículo 38. Becas, aquellas personas con discapacidad en cuya localidad no exista un establecimiento educativo público con servicios adecuados para atender a sus necesidades educativas especiales podrán recibir del Instituto Ecuatoriano de Crédito Educativo, becas y créditos educativos, a fin de que asistan a una institución educativa particular o fisco misional que sí ofrezca los servicios adecuados, de conformidad con la normativa específica que se expida para el efecto, Artículo 38, Becas para aquellas personas con discapacidad en cuya localidad no exista un establecimiento educativo público con servicios adecuados para atender a sus necesidades educativas especiales podrán recibir del Instituto Ecuatoriano de Crédito Educativo, becas y

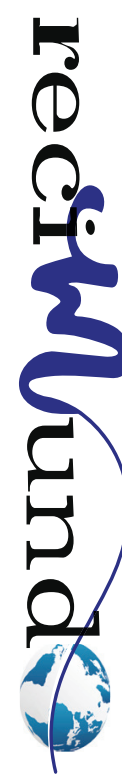


créditos educativos, a fin de que asistan a una institución educativa particular o fisco misional que sí ofrezca los servicios adecuados, de conformidad con la normativa específica que se expida para el efecto, la Secretaria Nacional de Educación Superior, Ciencia, Tecnología e Innovación hará cumplir a las instituciones de educación superior públicas y privadas la concesión de becas de tercer y cuarto nivel, en sus modalidades presencial, semipresencial y a distancia, para personas con discapacidad, aplicando criterios de equidad de género, Artículo 40, difusión en el ámbito de educación superior, la Secretaría Nacional de Educación Superior, Ciencia, Tecnología e Innovación, asegurará que en todas las instituciones de educación superior se transversalice el conocimiento del tema de la discapacidad dentro de las mallas curriculares de las diversas carreras y programas académicos, dirigidos a la inclusión de las personas con discapacidad y a la formación humana de las y los futuros profesionales. (Ley Orgánica de Discapacidades)

\section{(ONU, 2012)}

La Convención Internacional de Derechos de las Personas con Discapacidad es un tratado internacional que protege los derechos de las personas con discapacidad. La Asamblea General de las Naciones Unidas aprobó la Convención Internacional de Derechos el 13 de diciembre de 2006. Meses más tarde comenzaba el ciclo de firmas por parte de los países que se acogían al texto, en concreto, el 30 de marzo de 2007 , En total, el documento ha sido firmado por 82 países, y en España el texto entraba en vigor en mayo de 2008. España ratificó la Convención Internacional de Derechos el texto el 3 de diciembre de 2007, este texto legal pretende que los países firmantes asuman un compromiso de integración de las personas con discapacidad. Para ello se llevarán a cabo iniciativas orientadas a elaborar políticas y normativas que protejan los derechos que reconoce la convención, combatir prejuicios en contra de las personas con discapacidad, campañas de concienciación, los niños con discapacidad o padres con discapacidad, no podrán ser separados de sus padres ni de sus hijos, respectivamente, garantizar justicia e igualdad, protección de la integridad física y moral de las personas con discapacidad, garantía de que no serán explotados o sometidos contra su voluntad, Prohibir la discriminación, y un largo etcétera de medidas en pro de las personas con discapacidad y su integración. (Convención Internacional de Derechos de las Personas con Discapacidad)

\section{(Meritxell Solé, 2019)}

Este estudio se centra en el marco de un sistema educativo inclusivo. En los últimos años, se ha reforzado legislativamente la escuela inclusiva desde las diferentes políticas educativas promoviendo un cambio de mirada en el conjunto de la sociedad y de la comunidad educativa. Por lo tanto, surge la necesidad de implementar metodologías innovadoras para atender la diversidad de todo el alumnado. Y consideramos que la Educación Asistida con Animales puede ser una de ellas, por cuanto se ha demostrado los beneficios de la interacción humano-animal y su incidencia en factores como la socialización, la disminución del estrés, etc. Un sistema educativo inclusivo ofrece al alumnado aquellas medidas y soportes que son más adecuados para facilitar su participación y aprendizaje en un contexto educativo ordinario. Estas acciones y prácticas se dividen en universales, adicionales e intensivas. El estudio se ha basado en la metodología observacional sistemática aplicada a dos estudios de casos de niños que con diversidad funcional. Se ha planteado un diseño Mixed Methods incrustado o embedded design que pretende interrelacionar el enfoque cuantitativo con el cualitativo para obtener una visión amplia de los datos analizados. En este proceso se ha validado un sistema observacional de categorías Ilama- 
do OSAA (Observational System of Animal Assisted Interventions) generalizable en entornos ordinarios en los que se apliquen las Intervenciones Asistidas con Animales. A partir del análisis de los patrones de conducta (T-Patterns) y del análisis de contenido de las entrevistas validadas a los profesionales educativos que han participado en las sesiones de EAA, mediante el software Atlas.ti, se han constatado los beneficios que tiene la aplicación de la EAA en los niños con diversidad funcional de diferente etiología en un contexto educativo inclusivo, más concretamente, en un Apoyo Intensivo a la Educación Inclusiva (seis). Así pues, se demuestra que la EAA es una metodología eficiente de atención a la diversidad que aporta beneficios en la estimulación cognitiva, motriz, multisensorial, comunicativa y emocional en los niños como medida educativa que refuerza la adquisición de competencias curriculares. Estos beneficios surgen a partir de la interacción triádica entre el niño, el animal de terapia y el docente. La presencia del perro incrementa la producción de patrones comunicativos acompañados de expresiones de placer. Además, de una conexión atencional y motivacional más elevada del alumnado en la situación de enseñanza-aprendizaje (Solé, Meritxell (Fecha de defensa: 18-12-2019)

\section{(Martinez Ferrer, 2006)}

Desde que se aprobó, en 2004, la recomendación estratégica en la Asamblea Extraordinario del International Paralímpica Committe celebrada en El Cairo (Egipto) para favorecer la inclusión de los diferentes deportes Paralímpicos en las Federaciones Deportivas Internacionales (unideportivas), nos encontramos inmersos en una nueva situación en el deporte federado, representada por la inclusión del deporte para personas con discapacidad y sus modalidades deportivas en el seno de las federaciones de deportes específicos a nivel internacional y consecuentemente a nivel nacional. Este proceso ya se ha iniciado en España con varios procesos de inclusión / integración del deporte adaptado en varias federaciones unideportivas nacionales. Es objeto de este estudio doctoral proponer y seguir una estrategia metodológica de investigación-acción, que nos aproxime al conocimiento existente sobre la inclusión del deporte adaptado a las federaciones deportivas a través de sus gestores, técnicos y deportistas, comparando el conocimiento entre federaciones de deporte adaptado y de las federaciones unideportivas mediante la técnica participativa del "focus group", para poder proponer las bases de un modelo de intervención que sea útil para transformar su entorno en inclusivo, enmarcado en criterios consensuados de " buenas Prácticas Inclusivas ". A través de la metodología de la investigación-acción y del "focus group", en este estudio se analiza el conocimiento existente sobre los procesos de integración - inclusión del deporte adaptado a las federaciones unideportivas en España. Se comparan las creencias y las opiniones de personas representantes de las federaciones de deporte adaptado (pluriesportives) y las federaciones unideportivas. Esta tesis doctoral es una investigación imbricada y relacionada en sus contenidos con el proyecto Blanquerna Inclusión - BLiNCL, apoyada y subvencionada por el Consejo Superior de Deportes y el Comité Paralímpico Español (subvención: 021 / UPR10 / 12); para objetivar, evaluar y construir conocimiento sobre la inclusión en el deporte adaptado en España. Los participantes en el estudio representan 3 roles fundamentales en la organización deportiva: deportistas, técnicos y gestores. Las reuniones de "focus group", se han grabado en vídeo y audio, el contenido se ha analizado a través de una rigurosa metodología cualitativa de triangulación e informática a través del programa ATLAS.ti - The Qualitative Data Analysis Software, versión 2.4. y en base a los resultados obtenidos; realizándose una reflexión por parte de jueces expertos que han facilitado, a través de la discusión y las propuestas, el desarrollo de una propuesta

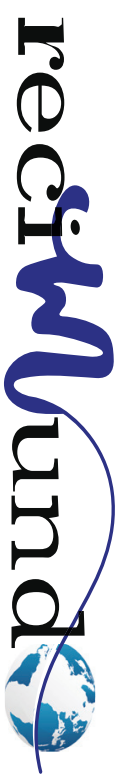


guía que ayude a la inclusión / integración en el deporte federado en España. El objetivo final del estudio es la elaboración de un "Protocolo inclusión del deporte de competición de las personas con discapacidad en las federaciones deportivas convencionales - unideportivas, en España" realizando propuestas razonadas y con base científica , inspiradas en los resultados del trabajo de campo, enriquecida con opiniones de expertos inclusivos y de expertos del deporte federado, para que puedan ser aprovechadas por las federaciones deportivas de España y de sus órganos rectores ( Martínez Ferrer, José Oriol (Fecha de defensa: 18-012016)

\section{Bibliografía}

Asamblea Constituyente. (2008). Constitución de la Republica del Ecuador. Quito: Lexis.

Asamblea Nacional. (2012). Ley Orgánica de Discapacidades. Quito: Lexus.

Martinez Ferrer, J. (2006). Estudio y protocolización del proceso de inclusión del deporte de competición de las personas con discapacidad en las federaciones deportivas convencionales en el Estado Español. Blanquerna, España: Universidad Ramon Llull. Obtenido de https://www.tesisenred. net/handle/10803/351962\#page $=1$

Meritxell Solé, R. (2019). L'Educació assistida amb animals com a mesura d'atenció a la diversitat a l'escola inclusiva. Facultat d'Educació, Psicologia i Treball Social. Lérida, España: Universitat de Lleida.

ONU. (2012). La convención internacional de derechos de las personas con discapacidad. Nueva York, Estados Unidos: Naciones Unidas.

\section{CITAR ESTE ARTICULO:}

Vásquez Aguilar, D., Soto Chávez, L., Allauca Amaguaya, M., \& Benitez Troya, F. (2020). Aprendizaje inclusivo para las personas con discapacidad. RECIMUNDO, 4(1(Esp)), 182-190. doi:10.26820/recimundo/4.(1).esp.marzo.2020.182-190

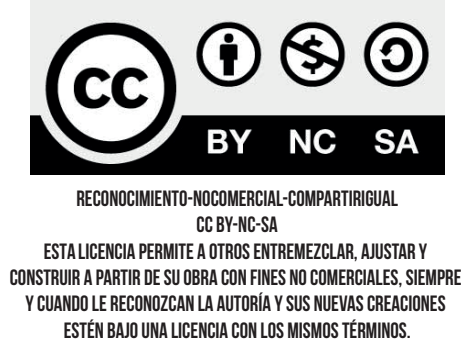

